\title{
Heart Failure Due to Cardiac Transthyretin Amyloidosis
}

\author{
Humberto Butzke da Motta, ${ }^{1(0)}$ Guilherme Oliveira Magalhães Costa, ${ }^{\circledR([)}$ Andreia Biolo $^{1,2(0)}$ \\ Serviço de Cardiologia, Hospital de Clínicas de Porto Alegre', Porto Alegre, RS - Brazil \\ Faculdade de Medicina, Universidade Federal do Rio Grande do Sul', Porto Alegre, RS - Brazil
}

\begin{abstract}
A 90-year-old male patient presented with complaints and physical examination compatible with decompensated heart failure. Electrocardiogram showed atrial fibrillation and low voltage in limb leads, and echocardiogram identified biatrial dilatation, in addition to mild increases in ventricular wall thickness and diameter. Magnetic resonance imaging confirmed suspicion of cardiac amyloidosis. With negative serum and urinary protein electrophoresis and positive pyrophosphate cardiac scintigraphy, diagnosis of transthyretin cardiac amyloidosis was confirmed. Transthyretin gene mutations were not identified on sequencing, characterizing wild-type transthyretin cardiac amyloidosis.
\end{abstract}

\section{Case Report}

A 90-year-old man sought emergency assistance at a hospital complaining of dyspnea on mild exertion, orthopnea, paroxysmal nocturnal dyspnea, and lower limb edema for 3 weeks. Until 4 months before presentation, he had been able to walk close to his residence without any major limitations and climb 2 flights of stairs. Since then, he had had a decline in functional capacity, with progressive dyspnea on exertion. He did not complain of chest pain, palpitations, or syncope.

Previously, he had been diagnosed with glaucoma, benign prostatic hyperplasia, and depression, for which he was receiving treatment with timolol and travoprost eye drops, finasteride $5 \mathrm{mg}$ daily, doxazosin $2 \mathrm{mg}$ in the evening, and escitalopram $10 \mathrm{mg}$ daily. In the past, he had undergone inguinal hernioplasty and appendectomy, as well as transurethral resection of the prostate. He denied tobacco or alcohol use. After the death of his wife, which had occurred 3 months before hospitalization, he lived with his daughter. He had not traveled recently, and he had no history of allergies.

On physical examination upon arrival at the hospital, he was in regular general state, with well hydrated and colored mucous membranes; he was anicteric, acyanotic, alert, oriented, and cooperative. Blood pressure was 107/62 mmHg; heart rate was $110 \mathrm{bpm}$, and respiratory rate 24 breaths per minute. He was afebrile, and peripheral oxygen saturation

\section{Palavras-chave}

Heart Failure; Amyloidosis; Prealbumin.

\footnotetext{
Mailing Address: Andreia Biolo •

Serviço de Cardiologia, Hospital de Clínicas de Porto Alegre. Rua Ramiro Barcelos, 2350, Sala 2060. Postal Code: 90035-903, Bairro Santa Cecília, Porto Alegre, RS - Brazil.

Email: biolo.andreia@gmail.com

Manuscript received September 15, 2021, revised manuscript September 26, 2021, accepted September 26, 2021.
}

DOI: https://doi.org/10.36660/abchf.20210024 was $93 \%$, while breathing spontaneously with an $\mathrm{O} 2$ nasal catheter at $3 \mathrm{~L} / \mathrm{min}$. He showed jugular turgescence at $45^{\circ}$, irregular cardiac auscultation, with 2 sounds, and normal heart sounds, without murmurs. Pulmonary auscultation revealed abolished vesicular murmur at the right base, with crackling rales up to the middle thirds bilaterally. Palpation of the upper right quadrant of the abdomen was painful, and he had edema with Godet's sign in the lower limbs extending to the area below the knees. Extremities were warm, and capillary refill time was adequate.

Anteroposterior chest radiograph was conducted, showing bilateral pleural effusion, which was greater on the right, and extensive interstitial infiltrate, predominantly in bases, compatible with the clinical hypothesis of decompensated heart failure. Electrocardiogram on arrival showed atrial fibrillation rhythm with frequent monomorphic ventricular extrasystoles, left bundle branch block, and low voltage in limb leads (Figure 1a). Laboratory tests demonstrated ultrasensitive troponin I of $59.1 \mathrm{pg} / \mathrm{mL}$ (reference value 0 to $34.2 \mathrm{pg} / \mathrm{mL}$ ) and brain natriuretic peptide of $1243.2 \mathrm{pg} / \mathrm{mL}$ (reference value 0 to $100 \mathrm{pg} / \mathrm{mL}$ ).

Transthoracic echocardiogram revealed dilation of both atria, slightly increased ventricular diameters, septal thickness of $13 \mathrm{~mm}$, and posterior wall thickness of $11 \mathrm{~mm}$, with left ventricular ejection fraction of $35 \%$ (Figure $1 \mathrm{~b}$ ). The right ventricle was normal, and there was moderate tricuspid regurgitation, with no signs of significant pulmonary hypertension. The other echocardiographic parameters are displayed in Table 1.

The clinical, electrocardiography, and echocardiography findings led to suspicion of cardiac amyloidosis. In order to define diagnosis, cardiac magnetic resonance and electrophoresis of blood and urine proteins with immunofixation were initially performed. Cardiac magnetic resonance showed partial nulling of the myocardial signal with extensive late enhancement of mesocardial and endocardial non-ischemic aspect, with circumferential involvement, predominantly in the basal portions, and additional involvement of the right ventricle and atria. These characteristics and distribution are associated with amyloidosis (Figure 2). Electrophoresis did not identify any monoclonal paraproteinemia.

The patient was treated for decompensated heart failure with a diuretic, which resolved the congestion; functional limitation persisted, compatible with New York Heart Association functional class III. Moreover, anticoagulation with rivaroxaban was initiated in order to prevent thromboembolic phenomena secondary to atrial fibrillation.

When the patient was discharged, exams for transthyretin (TTR) gene mutation and myocardial scintigraphy with pyrophosphate were forwarded. Thirty days later, when he returned to the outpatient clinic, he showed clinical 
Figure 1a

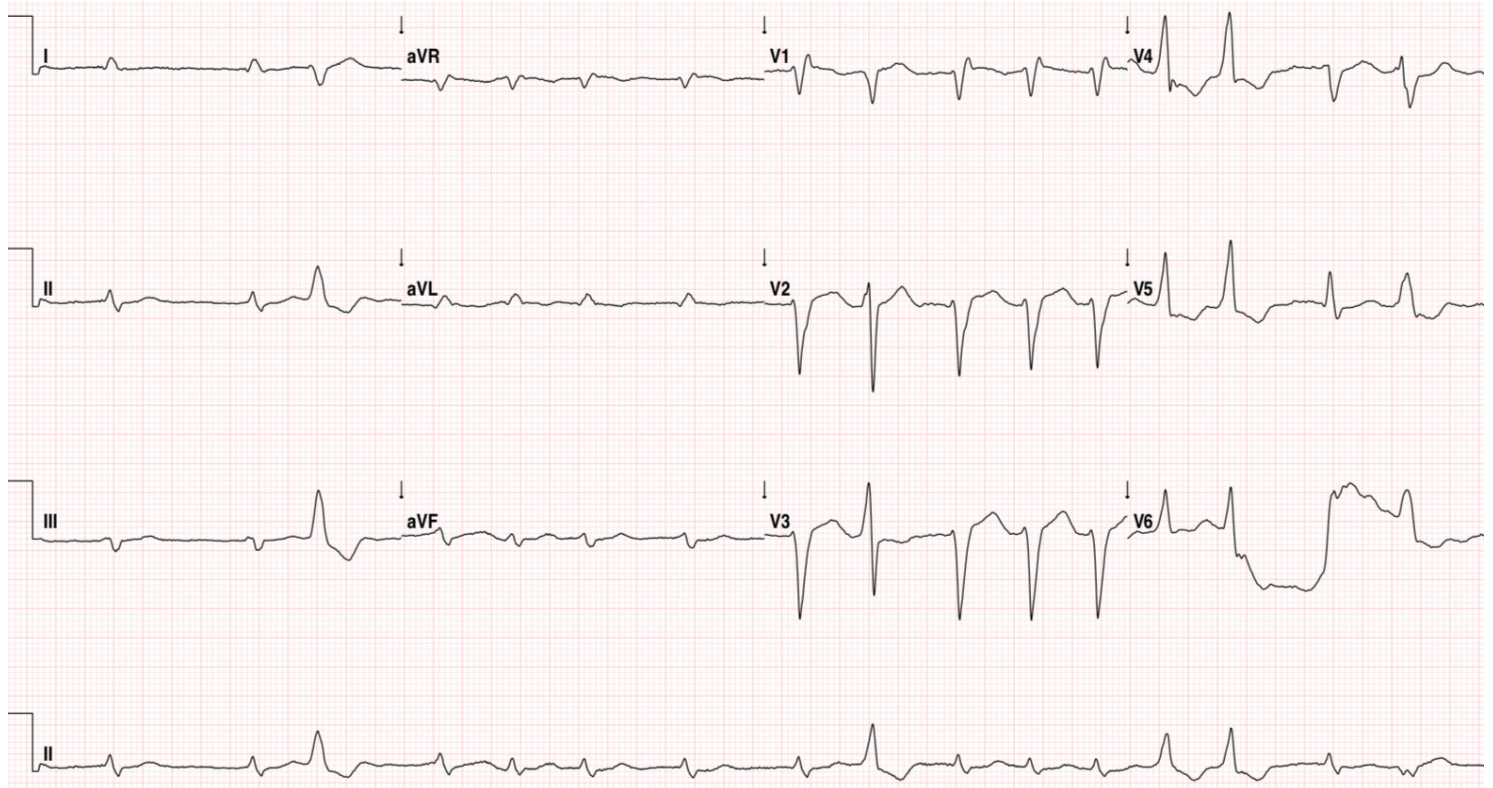

\section{Figure 1b}

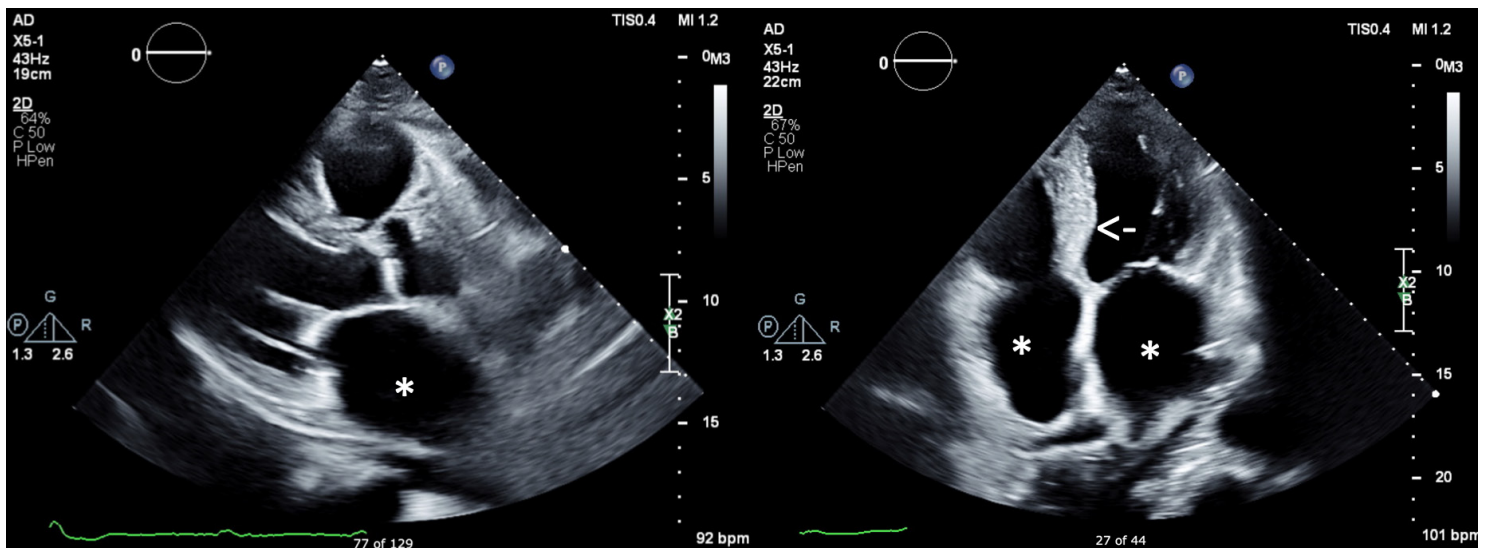

Figure 1 - 1a. Electrocardiogram demonstrating atrial fibrillation with frequent ventricular extrasystoles, left bundle branch block, and low voltage in limb leads. 1b. Transthoracic echocardiogram with biatrial dilatation $\left({ }^{*}\right)$ and increased thickness of the interventricular septum (<-).

Table 1 - Transthoracic echocardiogram

\begin{tabular}{lcc}
\hline Echocardiography measurements & & Reference values \\
\hline Aortic root & $37 \mathrm{~mm}$ & $58 \mathrm{~mm}$ \\
\hline Left atrial diameter & $72 \mathrm{~mL} / \mathrm{m} 2$ & up to 40 \\
\hline Left atrial indexed volume & $53 \mathrm{~mm}$ & up to $34 \mathrm{~mL} / \mathrm{m} 2$ \\
\hline Systolic left ventricle & $44 \mathrm{~mm}$ up to $59 \mathrm{~mm}$ \\
\hline Diastolic left ventricle & $13 \mathrm{~mm}$ & up to $40 \mathrm{~mm}$ \\
\hline Septal thickness & $11 \mathrm{~mm}$ & up to $11 \mathrm{~mm}$ \\
\hline Posterior wall thickness & $35 \%$ & up to $11 \mathrm{~mm}$ \\
\hline Left ventricular ejection fraction & $40 \mathrm{~mm}$ & up to $41 \mathrm{~mm}$ \\
\hline Basal right ventricle &
\end{tabular}




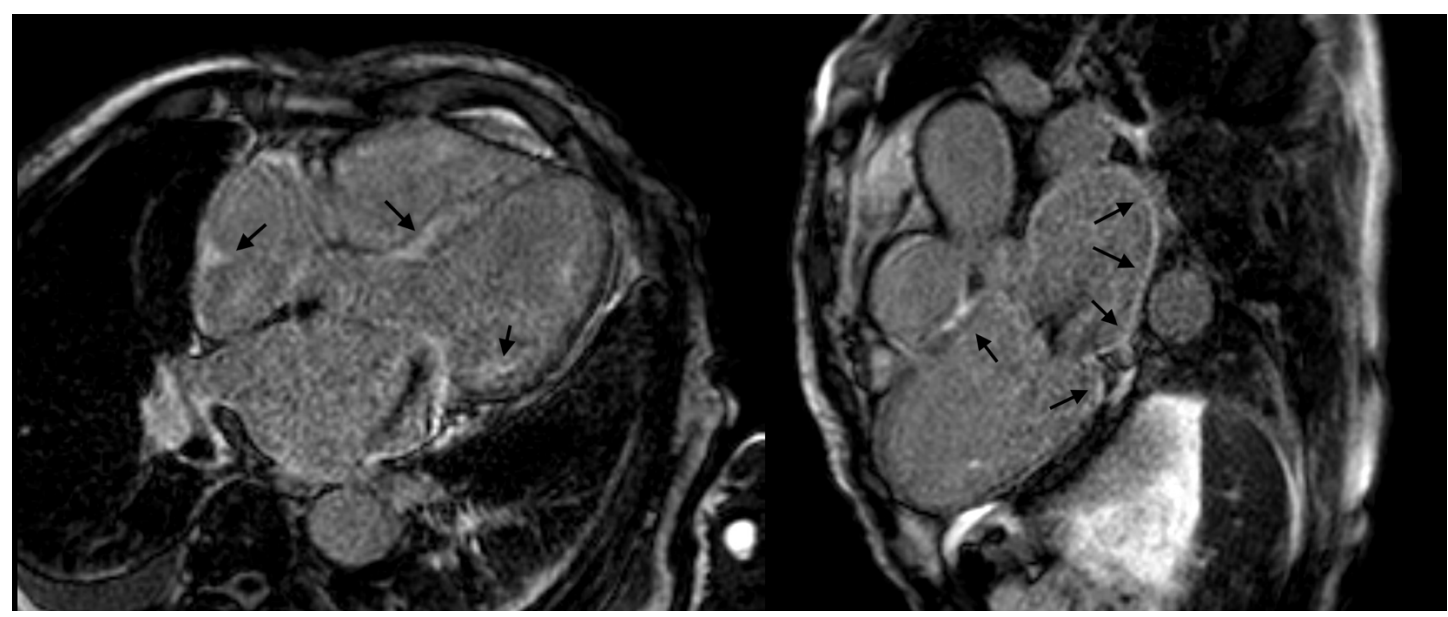

Figure 2 - Cardiac magnetic resonance imaging with delayed enhancement of non-ischemic appearance, predominantly in the basal portions of the left ventricle, right ventricle, and atria (arrows).

improvement, and he was able to take short walks, without orthopnea or clinical signs of pulmonary congestion. Investigation of mutations of the transthyretin gene was negative, and scintigraphy showed grade 2 myocardial uptake (Figure 3). These findings, in the absence of evidence of plasma cell dyscrasia, are compatible with the diagnosis of wild-type TTR amyloidosis. Thus, treatment with tafamidis $80 \mathrm{mg}$ a day was prescribed, and outpatient follow-up was maintained. The patient is waiting for the medication to be provided.

\section{Discussion}

Amyloidosis is a systemic or localized disease characterized by tissue deposition of insoluble fibrillar proteins that are resistant to proteolysis and that have lost their conformation, which can lead to organic dysfunction, including the heart. More than 30 types of proteins with amyloidogenic potential have been described. However, about $95 \%$ of cases of cardiac amyloidosis are related to 2 types of deposition: light chain immunoglobulins (AL) or transthyretin (ATTR); the latter may be secondary to mutation (ATTRm) or wild (ATTRwt), usually induced by changes related to aging. ${ }^{1}$ Although it is one of the most rapidly progressive heart diseases and one of the ones with the worst prognosis, with average untreated survival of less than 6 months for the AL form and between 3 and 5 years for ATTR, diagnosis often goes unnoticed or is delayed. It is estimated that the prevalence of $\mathrm{AL}$ amyloidosis is around 8 to 12 cases per million, and 30\% to $50 \%$ of these individuals will develop some cardiac symptom. The estimated prevalence of the ATTR form is more uncertain, with recent data suggesting underdiagnosis, especially in the elderly. Autopsy studies have shown amyloid TTR deposits in the myocardium of $25 \%$ of individuals over 80 years of age. ${ }^{1,2}$

Heart failure with preserved ejection fraction, especially in elderly men, is an early manifestation characteristic of cardiac amyloidosis, most often accompanied by echocardiographic changes (concentric hypertrophy, diastolic dysfunction, and septal thickening). ${ }^{2}$ Atrial fibrillation or flutter or conduction system abnormalities are also common findings. ${ }^{3}$ Bilateral carpal tunnel syndrome manifests approximately 5 to 10 years before diagnosis of ATTRwt amyloidosis in up to $70 \%$ of patients, and it may indicate an initial manifestation of transthyretin deposits. Sensory and autonomic neuropathy, orthostatic hypotension, biceps tendon rupture, spinal stenosis, and digestive disorders are also among the multisystem manifestations that may raise suspicion of ATTR. Therefore, with patients who complain of progressive exercise intolerance associated with any of these conditions and cardiac imaging suggestive of concentric thickening of the left ventricle, with an infiltrative phenotype or a restrictive pattern, it is necessary to be alert to the possibility of cardiac amyloidosis. ${ }^{2,3}$

Given that it is a disease with complex diagnosis, high clinical suspicion is fundamental. As highlighted in the recent Position Statement of the Brazilian Society of Cardiology ${ }^{4}$, there are some warning signs, also known as clinical clues, that should lead to further investigation. In the reported case, there was a considerable mismatch between the left ventricular concentric hypertrophy and the electrocardiographic manifestations, most notably in the limb leads.

When facing clinical suspicion of cardiac amyloidosis, careful analysis of cardiovascular tests is essential. Resting electrocardiograms may show suggestive changes, such as low voltage, absence of R-wave progression in precordial leads, or changes in heart rhythm. On echocardiography, we may identify characteristics of restrictive infiltrative cardiomyopathy, with diastolic dysfunction, thickening of the walls and septum, increased filling pressures, and biatrial dilatation. Ejection fraction usually remains preserved until later stages of the disease. Cardiac magnetic resonance imaging makes it possible to accurately assess changes in myocardial tissue, such as increased ventricular thickness, as well as the presence of delayed enhancement, which have a sensitivity of $85 \%$ and a specificity of $92 \%$ for diagnosing cardiac amyloidosis.

In order to differentiate between the AL and ATTR forms, a search for monoclonal light chains must be carried out, requiring the measurement of the serum kappa:lambda ratio and serum 


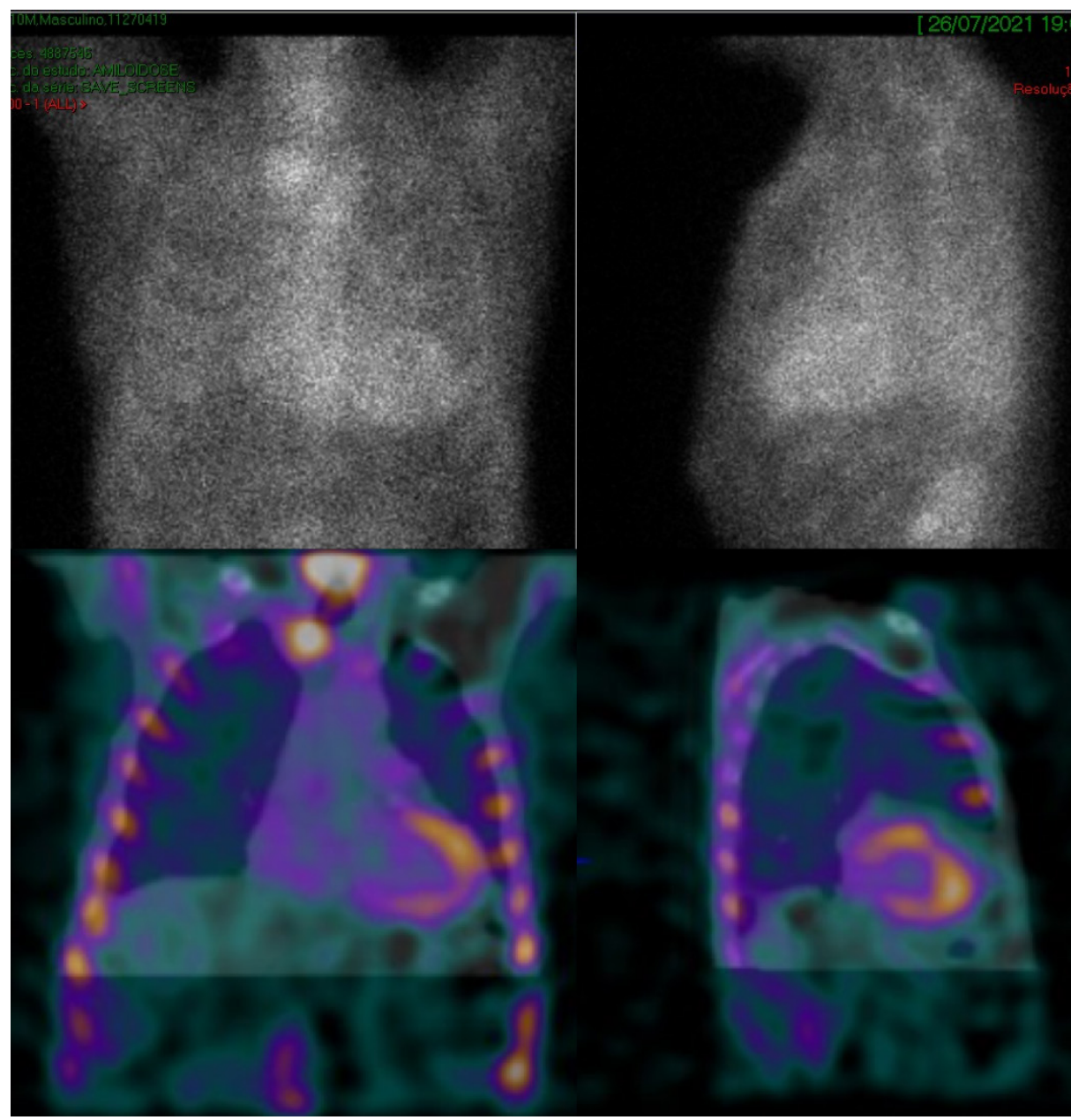

Figure 3 - Myocardial pyrophosphate scintigraphy with planar images (upper quadrants) and SPECT (lower quadrants), showing increased cardiac uptake in relation to the contralateral chest, with similar intensity to that of the costal arches (grade 2).

and urinary immunofixation. In the event that one of these tests is positive, a hematologist should be consulted, and a biopsy of peripheral tissues should be obtained for analysis and confirmation of diagnosis, as well as typing of the amyloid protein.

In the absence of monoclonal proteins, the cardiological path of the Brazilian Position Statement on Amyloidosis should be followed $^{4}$ (Figure 4). Cardiac scintigraphy with pyrophosphate is the exam of choice for confirming diagnosis, given that it can have $99 \%$ sensitivity and $86 \%$ specificity in diagnosing the ATTR form. Gene sequencing is recommended to differentiate between wild and hereditary forms.

Treatment of ATTR involves treating the clinical syndrome and specific approaches that interfere with the formation and deposition of amyloid fibrils. Liver transplantation was once the preferred option, but, currently, the use of TTR stabilizers, inhibitors of liver synthesis of TTR, and degradation and reabsorption of TTR fibrils has gained prominence. Among these strategies, tafamidis, a molecule that effectively inhibits the cascade of amyloid fibril formation, is the only drug that has been proven to prolong survival in patients with ATTR. ${ }^{5}$ Among therapies in the experimental phase, the following stand out: patisiran $^{6}$ (microRNA that inhibits expression of genes that encode the liver production of TTR), compounds that stabilize TTR, and compounds that induce TTR degradation and reabsorption.

\section{Acknowledgements}

We would like to thank Dr Gabriel Grossmann (Serviço de Medicina Nuclear, Hospital Moinhos de Vento, Porto Alegre, Rio Grande so Sul, Brazil), for generously allowing us to reproduce the scintigraphy images.

\section{Author contributions}

Conception and design of the research: Motta HB, Biolo A. Acquisition of data: Motta HB, Costa GOM, Biolo A. Writing of the manuscript: Motta HB, Costa GOM, Biolo A. Critical revision of the manuscript for intellectual content: Motta $\mathrm{HB}$, Biolo A. 


\section{Case Report}

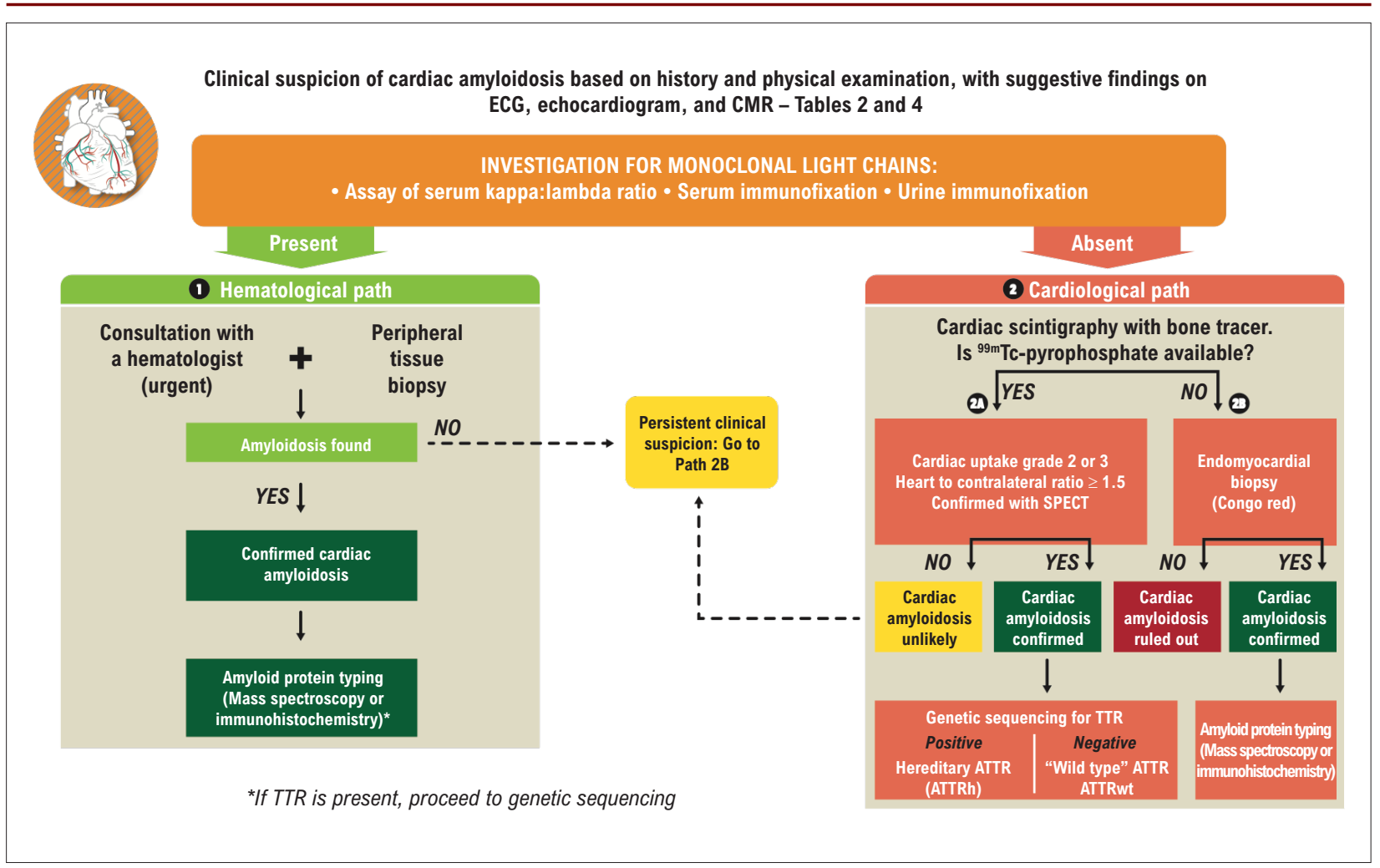

Figure 4 - Flowchart for diagnostic investigation of cardiac amyloidosis, according to the Position Statement of the Brazilian Society of Cardiology. ATTR: transthyretin amyloidosis; CMR: cardiac magnetic resonance; ECG: electrocardiogram; TTR: transthyretin

\section{Potential Conflict of Interest}

No potential conflict of interest relevant to this article was reported.

\section{Sources of Funding}

There were no external funding sources for this study.

\section{Study Association}

This study is not associated with any thesis or dissertation work.

\section{Ethics approval and consent to participate}

This article does not contain any studies with human participants or animals performed by any of the authors.

\section{References}

1. Maurer MS, Elliott P, Comenzo R, Semigran M, Rapezzi C. Addressing Common Questions Encountered in the Diagnosis and Management of Cardiac Amyloidosis. Circulation. 2017;135(14):1357-77. doi: 10.1161/ CIRCULATIONAHA.116.024438.

2. Dorbala S, Cuddy S, Falk RH. How to Image Cardiac Amyloidosis: A Practical Approach. JACC Cardiovasc Imaging. 2020;13(6):1368-83. doi: 10.1016/j. jcmg.2019.07.015.

3. Muchtar E, Dispenzieri A, Magen H, Grogan M, Mauermann M, McPhail ED, et al. Systemic Amyloidosis from A (AA) to T (ATTR): A Review. J Intern Med. 2021;289(3):268-92. doi: 10.1111/joim.13169.

4. Simões MV, Fernandes F, Marcondes-Braga FG, Scheinberg P, Correia EB, Rohde LEP, et al. Posicionamento sobre Diagnóstico e Tratamento da Amiloidose Cardíaca - 2021. Arq. Bras. Cardiol. 2021;117(3):561-98. doi: 10.36660/ abc. 20210718 .

5. Maurer MS, Schwartz JH, Gundapaneni B, Elliott PM, Merlini G, WaddingtonCruz M, et al. Tafamidis Treatment for Patients with Transthyretin Amyloid Cardiomyopathy. N Engl J Med. 2018;379(11):1007-16. doi: 10.1056/ NEJMoa1805689.

6. Adams D, Gonzalez-Duarte A, O'Riordan WD, Yang CC, Ueda M, Kristen AV, et al. Patisiran, an RNAi Therapeutic, for Hereditary Transthyretin Amyloidosis. N Engl J Med. 2018;379(1):11-21. doi: 10.1056/NEJMoa1716153. 
